Voix et Images

voixetimages

\title{
Entretien avec Carole Fréchette
}

\section{Hervé Guay}

Volume 39, numéro 1 (115), automne 2013

Théâtre et médias

URI : https://id.erudit.org/iderudit/1022990ar

DOI : https://doi.org/10.7202/1022990ar

Aller au sommaire du numéro

Éditeur(s)

Université du Québec à Montréal

ISSN

0318-9201 (imprimé)

1705-933X (numérique)

Découvrir la revue

Citer ce document

Guay, H. (2013). Entretien avec Carole Fréchette. Voix et Images, 39(1), 17-28.

https://doi.org/10.7202/1022990ar

Ce document est protégé par la loi sur le droit d'auteur. L'utilisation des services d'Érudit (y compris la reproduction) est assujettie à sa politique d'utilisation que vous pouvez consulter en ligne.

https://apropos.erudit.org/fr/usagers/politique-dutilisation/
Cet article est diffusé et préservé par Érudit.

Érudit est un consortium interuniversitaire sans but lucratif composé de l’Université de Montréal, l'Université Laval et l'Université du Québec à Montréal. Il a pour mission la promotion et la valorisation de la recherche. https://www.erudit.org/fr/ 


\title{
ENTRETIEN AVEC CAROLE FRÉCHETTE
}

\author{
$+++$ \\ HERVÉ GUAY \\ Université du Québec à Trois-Rivières
}

voix ET IMAGES Ce numéro de Voix et Images porte sur la relation qu'entretient le thêâtre québécois avec les médias de masse, comme les journaux, la radio, la télévision et Internet. Ce thème avait déjà été abordé à l'occasion dans votre thêâtre, mais il devient central dans votre dernière pièce, Je pense à Yu. Comme Madeleine, l'héroïne de ce drame, passez-vous beaucoup de temps sur Internet? Vous sentez-vous parfois interpellée ou submergée par les informations diffusées par les grands médias?

CAROLE FRÉCHETTE Je n'ai jamais calculé précisément le nombre d'heures que je passe sur Internet, mais j'imagine que $c^{\prime}$ est beaucoup. Je passe une grande partie de mes journées devant mon ordinateur et je fais des allers-retours constants entre mon travail en cours et la grande toile. En fait, comme la plupart des gens aujourd'hui, j'utilise Internet à plusieurs moments de la journée et pour toutes sortes de raisons : chercher la signification d'un mot, trouver une information précise sur un sujet quelconque, écrire un courriel, trouver un numéro de téléphone, lire les journaux, humer un peu de l'air du temps sur Facebook, etc. Est-ce que je me sens submergée par l'information? Absolument. C'est étourdissant. C'est une affirmation banale, mais tout à fait vraie. Il est stimulant d'avoir au bout des doigts tant d'information - on peut tout savoir en quelques secondes - , mais on est facilement noyé dans cet océan de données. Et je m'y noie souvent! Je ne sais pas où je me situe dans l'échelle d'utilisation d'Internet; je ne pense pas faire partie de ceux qui en sont accros. Je n'ai pas de blogue, pas de compte Twitter, je ne suis pas compulsive, mais il n'empêche qu'Internet occupe une grande place dans mes journées, et cette place va en grandissant, à mesure que je m'équipe d'appareils (téléphone intelligent, ordinateur portable très léger) qui me permettent de rester branchée partout et en tout temps.

voIX ET IMAGES Qu'est-ce qui vous a amenée à mettre Internet au centre de Je pense à $Y u$ ?

CAROLE FRÉCHETTE Je n'ai pas décidé de mettre les médias ou Internet au centre de ma pièce. C'est arrivé d'une façon toute naturelle. En lisant le journal, un matin de février 2006, je suis tombée sur un entrefilet de quelques lignes à propos d'un journaliste chinois, Yu Dongyue, qui venait de sortir de prison après dix-sept ans d'emprisonnement pour avoir lancé de la peinture sur le portrait de Mao Tsé-toung en 1989, au cours des événements de la place Tian'anmen. L'article disait que Yu Dongyue 
souffrait de maladie mentale à la suite des mauvais traitements qu'on lui avait infligés. Encore aujourd'hui, je ne sais pas pourquoi cette nouvelle m'a attirée. On lit tant d'histoires terribles dans les journaux. Pourquoi ai-je été si touchée par celle-ci? Peut-être à cause de la témérité du geste, de sa dimension sacrilège. Le matin même, je me suis demandé : est-ce que je me souviens de cet événement précis? J'avais suivi les manifestations de Tian'anmen à la télé comme tout le monde. Je suis allée à mon ordinateur et j'ai tapé Yu Dongyue sur Google. J'ai découvert que son cas était documenté par des organismes humanitaires comme Amnistie internationale. J'ai appris qu'il n'était pas le seul à avoir lancé de la peinture ce jour de mai 1989. Ils étaient trois jeunes hommes et tous les trois avaient fait de la prison. J'ai immédiatement pensé : un jour, je vais écrire quelque chose à ce sujet. J'ai mis l'article de côté, car j'étais en train de travailler à une autre pièce. Au bout de deux ans, j'ai relu l'entrefilet qui m'attendait, entre les pages de mon cahier, et j'ai été tout aussi attirée, fascinée, troublée par cette histoire. Je me suis alors demandé comment j'allais pouvoir m'en approcher. Il était évident pour moi que je n'allais pas faire de Yu Dongyue un personnage de ma pièce, que celle-ci n'allait pas se passer à Tian'anmen en 1989, car c'était une réalité beaucoup trop éloignée de moi. J'ai pensé que je devais partir de ma propre expérience, de mon propre rapport à cet événement survenu à l'autre bout du monde. C'est ainsi que j'ai eu l'idée d'écrire une pièce à propos d'une femme qui, comme moi, trouve dans un journal un article sur Yu Dongyue. Pour en savoir plus sur lui, elle fait ce que j'ai fait, ce que l'on fait tous : elle va à son ordinateur et tape son nom sur un moteur de recherche. C'est ainsi qu'Internet est entré dans ma pièce. Le personnage de Madeleine a pris forme lentement. Comme elle était en quelque sorte mon alter ego, il était évident pour moi qu'elle devait être une femme d'âge mûr, arrivée à un moment de la vie où on commence à faire des bilans. Elle s'est détachée de moi par la suite, à mesure que je lui ai inventé un passé, une histoire précise. J'en ai fait une militante, une femme de convictions, d'action, de combat, qui a cru au changement toute sa vie et se retrouve tout à coup en crise de sens. J'avais depuis longtemps envie d'écrire à propos des gens qui ont besoin de réponses radicales face à l'injustice et à tout ce qui ne va pas dans le monde. Madeleine a été une femme intransigeante et révoltée, ce qui est assez loin de moi, mais elle se trouve dans une espèce de passage à vide qui l'amène à poser un regard plus inquiet et incertain sur le monde, ce qui me ressemble tout à fait.

VOIX ET IMAGES Faites-vous une distinction entre Internet et les médias traditionnels? CAROLE FRÉCHETTE Internet ne ressemble pas aux autres médias, car il permet un accès instantané à une quantité phénoménale d'informations et il offre la possibilité d'entrer en profondeur dans chacun des sujets à travers les hyperliens. Je ne crois pas que j'aurais écrit Je pense à Yu s'il n'y avait pas eu Internet. J'aurais lu l'entrefilet dans le journal, j'aurais été intéressée et touchée de la même façon, mais pour aller plus loin, il m'aurait fallu me rendre à la bibliothèque, consulter de vieux journaux pour tenter de retracer l'histoire de Yu Dongyue, retrouver les articles de 1989, etc. Cela aurait été possible, évidemment, et peut-être que je l'aurais fait, mais la pièce aurait forcément été différente. La structure même de Je pense à Yu repose sur le type d'accès à l'information que permet Internet. Madeleine s'enfonce de plus en plus dans l'histoire de Yu. Elle commence par taper son nom, puis elle tape «Tian'anmen 1989 », puis elle 
tape le nom du dirigeant chinois qui a été à l'origine des manifestations, et ainsi de suite. La pièce est construite en avancées successives, «par hyperliens », dans l'histoire de Yu, mais aussi dans le passé de Madeleine, de Lin et de Jérémie.

VoIX ET IMAGES Vous venez d'inaugurer un site Internet sur vous et votre œuvre. Qu'est-ce qui vous a guidée dans sa mise en forme? Peut-on comparer la construction d'un tel site à l'écriture d'une pièce ou d'un texte?

CAROLE FRÉCHETTE Longue histoire que ce site Internet: $j^{\prime} y$ pense depuis trois ans. Il existe pour deux raisons. D'abord, c'est une vitrine. Pour un artiste, il est important aujourd'hui de faire sa propre promotion sur Internet. Cela représente tout un travail, qui n'existait pas il y a une dizaine d'années. Il ne suffit pas d'aligner les faits, il faut aussi soigner la présentation, donner une image de soi-même qui nous semble juste, se mettre en scène en quelque sorte. En ce sens, c'est une petite création, même si ça n'a rien à voir avec l'écriture. Dans mon cas, comme une bonne partie de ma carrière se passe à l'extérieur du Québec et n'est pas nécessairement connue ici - je n'alerte pas les journaux chaque fois que j'ai une production à l'étranger -, il m’a semblé particulièrement intéressant de rendre disponibles ces informations. La seconde raison, c'est que ce site me permet de rassembler toutes les données concernant mes activités professionnelles - mes publications, les mises en scène, passées et présentes, de mes pièces, les articles sur mon œuvre, etc. Cela a été un effort énorme de réunir et d'organiser tous ces éléments répartis sur vingt-cinq ans d'écriture, mais, maintenant, tout est sur le site et je peux dire à ceux qui veulent en savoir plus sur mon parcours et mes actualités de le consulter. C'est très pratique. Reste à voir si j'aurai la discipline de mettre tout cela à jour régulièrement. Je dois dire que je n'aime pas du tout m'astreindre à ce travail d'archivage. J'éprouve aussi un certain malaise à me mettre ainsi en évidence. Je cherche donc toujours le bon équilibre : il n'y a pas de honte à mettre ce qui nous arrive en valeur. Mais il y a aussi beaucoup de vanité dans Internet. C'est un lieu inconfortable, malaisé à occuper.

VoIX ET IMAGES Mais il n'y a pas qu'Internet dans Je pense à Yu, puisque l'élément déclencheur vient d'un entrefilet publié dans la presse. Pour un auteur dramatique, dans quelle mesure les grands médias alimentent-ils l'écriture?

CAROLE FRÉCHETTE Les médias alimentent l'imaginaire comme tout le reste. Ils font partie de ce que nous absorbons chaque jour. Je suis influencée par les journaux, bien sûr. J'en lis tous les jours et je suis très intéressée par l'actualité. Mais je suis aussi influencée par les gens qui m'entourent, par ce que je vois dans la rue, par les petits événements du quotidien. Les médias, c'est le monde extérieur qui vient à soi. L'un des thèmes récurrents de mon écriture est la recherche de sa place dans le monde. Marie, Simon, Hélène, Justine, l'héroïne de la pièce que j'écris en ce moment, sont tous un peu en marge du monde. Ils sont conscients de ne pas être au cœur de son tourment. Je suis moi-même très consciente du lieu d'où je parle : l'Amérique du Nord, parmi une minorité francophone inquiète de son identité, au sein d'un grand pays paisible. À l'échelle de la planète, je fais partie d'un petit ensemble privilégié et je perçois les secousses qui agitent les peuples depuis un endroit relativement confortable. Les médias, les journaux et Internet sont des fenêtres sur le monde. On en trouve bien sûr des traces dans ce que j'écris, quoique ce soit loin d'être le sujet principal de mon œuvre. 
VOIX ET IMAGES La première allusion aux médias dans votre théâtre se trouve dans Baby Blues, votre première pièce. Des femmes ont regardé un film qui les a fait rire à la télévision, et Armande a vu un reportage sur la Floride: «Les marécages, les arbres pliés, c'était sauvage, on aurait dit la jungle. J'aurais pas cru... en Floride ${ }^{1}$.» La même Armande s'exclame plus loin : «Il a beaucoup neigé cette nuit. Il paraît qu'on a battu des records. J'ai entendu ça à la radio ${ }^{2}$.» Est-ce à dire que la télévision a été pour vous le premier média marquant? Et si tel est le cas, est-ce la fiction ou l'information qu'on y diffusait qui vous a le plus marquée?

CAROLE FRÉCHETte Dans mon histoire personnelle, la télévision a été le premier média d'importance, puisque je l'ai regardée bien avant de lire les journaux. Comme tout enfant. C'est très certainement la fiction qui m’a d'abord passionnée. Quand j'étais petite, j'adorais les séries comme Le comte de Monte-Cristo, Robin des Bois, Thierry la Fronde. Je me rappelle encore mon bonheur, le dimanche soir à dix-neuf heures, quand l'émission commençait! C'étaient des moments privilégiés. Contrairement à aujourd'hui, où l'on peut regarder tous les films et toutes les séries que l'on veut à tout moment, à cette époque, si on ratait un épisode, c'était fini. On ne pouvait pas le revoir avant dix ans au moins! La télévision et les bandes dessinées (j'ai été une grande fan de Tintin) ont été mes premières entrées dans la fiction. Je suis venue au roman plus tard, vers dix-sept, dix-huit ans. J'étais aussi fascinée par le cinéma, mais, quand j'étais enfant, je n'y allais pas souvent. J'ai un souvenir mémorable d'un film, La famille Trapp, ancêtre de La mélodie du bonheur. La mère d'une amie avait promis de nous y emmener. Chaque samedi, on attendait, mais elle changeait sans cesse d'idée. J'en ai rêvé pendant des semaines. Je crois que l'on peut voir l'influence de ces fictions dans mon thêâtre: leur ligne narrative très simple s'est imprimée en moi. voix et IMAGes Dans Les quatre morts de Marie, vous citez les films de Walt Disney, et Marie a été marquée après avoir vu à la télévision des images de moines bouddhistes ayant «fait ça ${ }^{3}$ » [l'auto-immolation]. Marie raconte aussi avoir fait carrière à la télévision, comme fille montrant à l'écran les boules gagnantes au cours des tirages de la loterie nationale, carrière à laquelle elle met fin en croquant le numéro 8. C'est un contraste frappant que vous présentez ici entre le monde du divertissement et celui de l'action politique auxquels les médias donnent accès.

CAROLE FrÉCHETte Je n'y ai pas pensé en écrivant ces deux anecdotes, mais il est vrai qu'à l'époque, la télévision constituait pour moi à la fois une fenêtre ouverte sur le réel, sur la souffrance du monde, et un voile, un écran de superficialité qui cache cette souffrance derrière des divertissements anodins et souvent abrutissants. Et Marie est touchée par ces deux aspects, déchirée entre la conscience et l'oubli.

VoIX ET IMAGES L'actualité est aussi très présente dans votre thêâtre. Dans Les quatre morts de Marie, son père Théo dit être allé « [d]ans des villes avec des ouragans, des révolutions, des enfants qui meurent dans les rues ${ }^{4}$ ». On songe au Collier d'Hélène ${ }^{5}$,

1 Carole Fréchette, Baby Blues, Montréal, Les Herbes rouges, coll. «Théâtre», 1989, p. 37.22 Ibid., p. 47.

3 Carole Fréchette, Les quatre morts de Marie, Montréal, Les Herbes rouges, coll. "Théâtre», 1995, p. 72.4 Ibid., p. 45. 5 Carole Fréchette, Le collier d'Hélène, Carnières-Morlanwelz, Éditions Lansman, coll. «Théâtre à l'affiche», 2002, 43 p. 
pièce où vous évoquez les conséquences de la guerre civile libanaise. Aurions-nous une aussi grande conscience de ce qui se passe sur la scène internationale sans les médias?

CAROLE FRÉCHeTte Non, bien sûr. Sans les journaux, la radio, la télé, Internet, nous ne saurions pas grand-chose des guerres, des luttes politiques, des catastrophes naturelles qui sévissent d'un bout à l'autre de la planète. Il est difficile d'imaginer ce que serait notre vie sans cette connaissance immédiate de tout ce qui agite le monde. Il m'arrive souvent de penser que nous avons beaucoup trop d'information sur des événements qui ne nous affectent pas directement et que nous ne savons pas comment absorber tous ces faits. Nous avons accès à des bribes de réalité qui nous bouleversent, nous choquent, provoquent en nous un sentiment d'impuissance. Nous nous y arrêtons brièvement, puis nous continuons notre journée. Je suis persuadée que nous gardons quelque part sur notre disque dur personnel une trace diffuse de tous les malheurs qui nous sont racontés, de toutes les horreurs qui nous sont montrées, de tous les événements cocasses, insolites, heureux qui défilent sur nos écrans. Il n'est pas étonnant que ces images ressurgissent d'une façon ou d'une autre dans l'écriture.

VoIX ET IMAGES Les médias se font aussi sentir dans Les sept jours de Simon Labrosse. C'est grâce à une petite annonce que Simon trouve Nathalie, et il repère dans une revue sur le cerveau la clinique où pourrait être opéré Léo. En outre, Nathalie tient absolument à montrer la vidéocassette de son intérieur, comme si c'était un film digne de l'interêt du grand public. Enfin, avec son format en sept jours, la pièce rappelle une série télévisée. Dans son esthétique même, la pièce table sur le besoin de montrer aux autres, sous la forme d'un spectacle, ce qu'est une vie ordinaire. On dirait bien que vous voyez des côtés positifs au fait de se donner en spectacle, à défaut de souscrire aux objectifs de la «société du spectacle » que dénonçait Guy Debord. D’où vous est venue l'idée que Simon ait ce besoin ou encore veuille devenir spectateur de la vie des autres?

CAROLE FRÉCHETTE Comme toujours, la pièce a pris sa source dans une toute petite chose. Un jour, je devais avoir une discussion difficile avec un ami et cela me terrifiait. J'ai alors pensé : je paierais bien quelqu'un pour avoir cette discussion à ma place. Je me suis mise à imaginer un personnage qui offrirait ce genre de services à la population, quelqu'un qui prendrait les risques émotifs à votre place: un cascadeur émotif. Puis j'ai imaginé d'autres services qu'il pourrait proposer : spectateur personnel, finisseur de phrases, etc. À l'époque, le Centre des auteurs dramatiques avait lancé un appel de textes humoristiques en vue d'une soirée de lecture de courtes pièces. Je m'étais dit «moi, je ne suis pas drôle du tout», et j'avais jeté l'invitation au panier. Puis, j'ai pensé à mon cascadeur émotif, et je me suis dit qu'il avait un potentiel comique. J'ai donc écrit trois sketches sur trois services très spéciaux offerts par un garçon paumé. C'est ainsi qu'est né Simon Labrosse. Mes sketches ont eu beaucoup de succès et les gens m'ont encouragée à développer mon idée. Pour en faire une pièce, il me fallait trouver une structure qui relierait tous les métiers de Simon. J'ai décidé qu'il allait proposer au public le récit de ses sept tentatives pour vendre à ses contemporains ses services incongrus. Voilà comment est venue l'idée d'un personnage qui se donne en spectacle. C'était pour Simon une stratégie de survie. J'ai écrit Simon Labrosse à une période où je venais de quitter un travail bien rémunéré pour 
devenir une auteure à temps plein. Ayant décidé de me consacrer à l'écriture, je ressentais un besoin urgent d'exister comme auteure dans ma société. Sans m'en rendre compte, j'ai transposé dans cette pièce ma propre urgence de me tailler une place. Derrière l'acharnement de Simon à proposer ses idées formidables, il y avait moi, l'auteure, qui disait au public: si vous ne voulez pas de cette pièce, je vais vous en écrire une autre, et encore une autre, jusqu'à ce que vous en acceptiez une!

VoIX ET IMAGES Simon déclare à un certain moment: «Votre problème de fond, c'est que vous êtes toujours à l'arrière-plan ${ }^{6}$.» Selon vous, les médias ont-ils une responsabilité dans le fait que la vie des gens ordinaires compte de moins en moins aux yeux des autres? Est-ce la meilleure réponse au système de vedettariat instauré par les médias que d'assurer son autopromotion pour être certain d'être vu?

CAROLE FRÉCHETTE Je ne sais pas si les gens ordinaires ont moins d'importance à cause des médias. Il y a la vie que nous vivons et il y a le regard posé sur elle par la société. Ce n'est pas parce qu'une vie n'est pas médiatisée qu'elle n'existe pas, qu'elle n'a pas ses grandeurs et ses accomplissements, ses bonheurs et ses drames. Qu'est-ce que ça veut dire, «une vie moins importante»? Moins importante parce que non regardée publiquement? Même si on y porte peu d'attention, ces vies se déroulent et sont importantes pour tous ceux qui sont touchés par elles. Il y a en ce moment, c'est vrai, un immense besoin du regard public sur nos vies. Ce n'est pas nouveau. Dans les années 1960, Warhol disait déjà que tout le monde aurait ses quinze minutes de gloire à la télé. Avec les nouveaux médias, cette accession à une existence publique est facilitée. À travers Facebook, chacun peut attirer l'attention, trouver une façon de montrer ses bons coups, de paraître drôle, intelligent, sensible, conscientisé socialement, etc. Chaque entrée sur Facebook est une petite construction, une mise en scène de soimême. Chacun, qu'il soit artiste ou non, peut devenir une vedette sur la petite scène des médias sociaux. Le courant de la téléréalité qui s'est répandu au cours des dix dernières années va dans le même sens. Il me semble que le grand projet de la télé en ce moment tourne entièrement autour du vedettariat. D'une part, on utilise les vedettes à toutes les sauces, dans les émissions sportives, littéraires, politiques, etc., pour montrer que ces personnalités publiques sont au fond des gens ordinaires, comme nous. Et, d'autre part, on permet à des gens ordinaires de devenir des vedettes à travers la téléréalité. Dans un cas comme dans l'autre, l'important est le concept de "personnalité spéciale», digne d'attention, qui se détache de la masse... comme si le regard public procurait un «supplément d'être».

voIX ET IMAGES L'un des motifs importants de votre théâtre est d'ailleurs que vos héroïnes, en particulier, veulent être vues. Est-ce le propre d'une culture médiatique d'engendrer un désir insatiable d'être vu, peut-être parce que le regard n'est plus vraiment tourné vers l'autre, mais vers un écran qui attire tous les regards, vers des médias qui décident qui mérite d'être vu et qui ne le mérite pas?

CAROLE FrÉCHETTE C'est vrai. Les quatre morts de Marie commence par les mots « Je vous en prie/Regardez-moi ${ }^{7}$ ", que Marie adresse au public. Cependant, il n'y a pas,

$+++$

6 Carole Fréchette, Les sept jours de Simon Labrosse. Si sa vie vous intéresse, Montréal/Arles, Leméac/Actes sud, coll. «Actes sud - Papiers», 1999, p. 20. 7 Carole Fréchette, Les quatre morts de Marie, p. 11. 
dans cette prière, de désir de glamour médiatique. Marie est en quête d'elle-même. Elle a besoin du regard de l'autre pour comprendre qui elle est. Elle n'interpelle pas la société, elle s'adresse aux individus présents devant elle. Elle leur dit : «[E]nveloppezmoi,/soufflez sur moi ${ }^{8}$.» Elle cherche la chaleur et la vérité de leur regard.

voIX ET IMAGES Vous associez parfois les médias au prêt-à-penser, au conformisme : à défaut d'avoir une opinion, certains de vos personnages ont déjà lu ceci dans une revue, vu cela à la télévision, entendu ceci ou cela à la radio. Est-ce pour vous un effet inquiétant des médias?

CAROLE FRÉCHETTE Il est vrai que ces formules reviennent sans cesse dans mes pièces. Béatrice dit constamment, par exemple, qu'elle a beaucoup lu sur l'amour. Selon moi, elle n'a pas lu les bons livres! Enfin, passons. Mais je n'ai pas utilisé ces expressions dans le but de faire la critique d'un certain conformisme. Les informations glanées dans les médias sont conservées pêle-mêle dans notre mémoire et nous les ressortons à tout moment. Nous sommes constitués de tout ce que nous avons fait, mais aussi de ce que nous avons lu, de ce que nous avons regardé à la télé et au cinéma. Nous portons en nous cette somme de connaissances, certaines approfondies, d'autres bien superficielles, et nous y faisons référence constamment. Quand mes personnages disent «j'ai lu ça quelque part», ils font ce que nous faisons tous plusieurs fois par jour: citer un bout d'article, une nouvelle entendue à la radio, pour enclencher une discussion, pour réfléchir sur un sujet, rebondir sur un autre, etc. Ces bribes d'information alimentent notre pensée. On les transforme, on les réinterprète selon nos besoins.

VoIX ET IMAGES Même une pièce qui semble plus éloignée de l'actualité comme Jean et Béatrice trouve sa source dans une affiche posée dans les rues, ancêtre des médias actuels, sorte de haut-parleur. C'est cette affiche qui, à bien des égards, fait office de petite annonce et permet la rencontre des deux personnages. Et tant Béatrice que Jean paraissent sensibles à des aspects du monde que l'on associe volontiers aux médias. Béatrice prend en photo - "pour [s]es archives ${ }^{9}$ » - tous les hommes désireux de la séduire dans le but d'obtenir une prime substantielle, tandis que Jean «cherche dans le journal les photos des jeunes filles disparues, les portraits-robots des maniaques à la scie ${ }^{10}$ ». Béatrice prétend plus tard avoir été conçue alors que ses parents «regard[aient] les images d'un ouragan aux nouvelles de dix heures ${ }^{11}$ ». $\bar{A}$ première vue, on pourrait croire que, dans vos pièces, même les personnages proches du conte évoluent dans une société de l'image et ne peuvent échapper au sensationnalisme d'une certaine presse axée sur les faits divers et les désastres naturels. Est-ce exact?

CAROLE FRÉCHETTE Tous mes personnages vivent dans une société de l'image et ils font tous allusion à des choses qu'ils ont vues dans les médias. Ils sont exposés au sensationnalisme, certes, mais aussi à des informations plus approfondies. En faisant ces allusions aux images diffusées à la télé, je ne cherche pas à dénoncer le sensationnalisme des médias. Je n'écris jamais pour dénoncer, d'ailleurs. Je m’attache à

$$
++
$$

8 Ibid., p. 11. 9 Carole Fréchette, Jean et Béatrice, Montréal/Arles, Leméac/Actes sud, coll. «Actes sud - Papiers", 2002, p. 9.10 Ibid., p. 16.11 Ibid., p. 47. 
faire vivre des personnages, à regarder et à ressentir le monde à travers eux. J'essaie de ne pas juger. Les références à des images ou à des infos de la télé sont nombreuses dans mes pièces parce qu'elles font partie de notre réalité quotidienne. Nous baignons dans ces images jour après jour, nous les recevons, nous les transformons et les réinterprétons, elles ponctuent nos vies, nous sommes complètement traversés par elles. Et ces informations sont de toutes natures, certaines étant intellectuellement stimulantes, d'autres, superficielles, ridicules ou émouvantes, et tout cela s'imprime en nous de façon non discriminée. Nous serions des êtres très différents si nous vivions dans la forêt, coupés de ce flux médiatique. Je ne peux pas imaginer ce que j'écrirais si j'étais dans une telle situation...

voIX ET IMAGES Dans Jean et Béatrice, vous faites aussi le lien entre le métier d'acteur et la publicité. Béatrice précise qu'elle a été courtisée par un acteur connu qui «jouait le petit pois dans une annonce de macédoine ${ }^{12}{ }$. Ce portrait ressemble étrangement à celui de Marie, qui prête son corps à des tirages de loterie. N'est-ce pas aussi un effet pervers des médias, au Québec du moins, d'obliger les artistes à vendre n'importe quel produit pour gagner leur vie? Le déplorez-vous?

CAROLE FRÉCHETTE Nous abordons ici un autre débat: celui de la condition des acteurs au Québec. Les acteurs d'ici n'ont pas le choix de participer à ce grand cirque médiatique d'une façon ou d'une autre. Dans les années 1970, quand j'ai été formée comme actrice, il y avait un fort mouvement de responsabilisation de l'acteur. C'était l'époque de la création collective. Beaucoup de comédiens autour de moi - et j'ai fait partie du mouvement - refusaient d'être de simples marionnettes qui allaient mettre leur talent au service de projets discutables, à la télé ou au thêâtre. Un acteur de théâtre, à nos yeux, devait se sentir responsable de la parole qu'il prenait en public, à la scène comme au petit écran. Cette notion me semble avoir disparu aujourd'hui. Il est maintenant généralement accepté que les acteurs utilisent leur talent et leur notoriété pour vendre divers produits. J'imagine que certains acteurs se posent, individuellement, des questions morales ou éthiques au sujet de ce qu'on veut leur faire vendre. Mais il n'y a pas, à ma connaissance, de débat public, dans le milieu, à ce sujet. La réalité du théâtre est ainsi faite au Québec qu'un acteur ne peut pas gagner sa vie uniquement grâce aux arts de la scène. Et il n'a pas toujours le loisir de refuser un rôle dans une série qu'il juge de piètre qualité. Il doit même, éventuellement, assurer la promotion de cette série: participer à des émissions de cuisine, à des talk shows, à des quiz, etc. Nous sommes dans une société où le vedettariat est incroyablement développé, et la télévision est construite autour de ce système. Dans cette dérive, les acteurs perdent parfois le lien avec leur premier métier et deviennent des amuseurs publics. Au Québec, le milieu est petit, et tout est lié: tu ne veux pas refuser un rôle à la télé parce que tu risques de ne plus avoir d'offre pendant un certain temps. De plus, tu as avantage à travailler à la télé si tu veux jouer au théâtre, car les directeurs artistiques privilégient les acteurs connus du petit écran pour attirer un plus large public. Je comprends bien la situation, mais je trouve quand même dommage qu'il n'y ait pas, au sein de la profession, de réelle réflexion sur le métier d'acteur aujourd'hui.

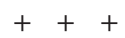

12 Ibid., p. 14. 
voix ET IMAGES Dans La petite pièce en haut de l'escalier, on parle peu des médias, mais le drame est centré sur l'imaginaire de la princesse, distillé par ceux-ci à l'adresse des jeunes filles sensibles, trop sensibles. Est-ce un élément de cette culture médiatique qui vous fait tiquer? Y en a-t-il d'autres?

CAROLE FRÉCHETte Beaucoup de phénomènes me tombent sur les nerfs dans les médias, mais je suis particulièrement irritée par la culture du paraître. La petite pièce en haut de l'escalier porte sur le mythe du beau mariage entre une belle jeune femme et un homme très riche. C'est une relecture du conte La barbe bleue transposé dans le monde d'aujourd'hui. La jeune femme qui a fait un mariage de rêve ressent malgré tout un manque, mais sa mère lui dit: «Tu as tout ce dont une jeune femme peut rêver : le mari extraordinaire, la maison, la piscine, les jardins. Qu'est-ce que tu veux de plus ${ }^{13}$ ? " Nous sommes dans la culture du succès, de la possession, de la consommation, de la jeunesse (plutôt: de l'apparence de jeunesse) et d'une certaine beauté désincarnée. Dans les médias, les femmes n'ont plus de vrai visage. Photoshop fait des ravages dans les magazines, et même dans certains programmes de théâtre où les portraits des acteurs sont tellement trafiqués qu'on a l'impression de visages de cire. Je trouve cette culture absolument terrible. Pour une femme, il est presque interdit de vieillir à la télévision. Si j'étais encore actrice, je trouverais très souffrant d'avoir à charcuter mon visage pour le rendre conforme aux standards de beauté actuels.

VoIX ET IMAGES Dans votre théâtre, une réelle tension existe entre confort et compassion; plusieurs de vos héroïnes sont mues par cette tension qui oppose une possible insouciance et la conscience d'inégalités, d'injustices à combattre. Dans quelle mesure les médias jouent-ils le rôle de courroie de transmission de ces attitudes?

CAROLE FrÉCHETte Les médias proposent un véritable tourbillon de superficialité, de confort et d'oubli, mais ils offrent aussi un accès à la souffrance du monde. Cette souffrance n'est pas seulement à l'autre bout de la planète, elle peut être dans la rue juste à côté de chez soi. Mes personnages ont une part d'eux-mêmes qui désire être touchée par la souffrance, qui éprouve de la compassion. Mais ils ne cherchent pas tellement à changer le monde. Dans Je pense à Yu, il est question de trois garçons qui ont voulu secouer le système rigide de leur pays. Ils ont pris pour ce faire un moyen extrêmement radical et risqué. Mais la pièce ne les glorifie pas et ne dit pas : voilà ce qu'il faut faire. Elle présente leur histoire avec beaucoup de respect et s'interroge sur le sens de leur action. Pour ma part, je suis ambivalente face à ce geste. J'admire la force et le courage qu'il a fallu pour l'accomplir, et je suis convaincue que le monde a parfois besoin de ces actes sacrificiels pour avancer. Mais il y a une autre partie de moi qui se demande, comme le personnage de Jérémie: "Qu'est-ce qui change vraiment?» L'humain reste l'humain. Un régime en remplace un autre, mais les luttes de pouvoir n'arrêtent jamais, ni la violence. Je suis toujours à la jonction de ces deux attitudes. C'est pourquoi même s'ils sont conscients de la souffrance du monde, mes personnages ne sont pas tellement dans des dynamiques de combat ni dans l'espoir de lendemains

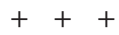

13 La réplique est citée de mémoire et correspond aux premières observations de la mère dans la pièce. Carole Fréchette, La petite pièce en haut de l'escalier, Montréal/Arles, Leméac/Actes sud, coll. "Actes sud - Papiers ", 2008 , p. 5. 
qui chantent. Un écrivain transmet avant tout son rapport au monde. Ce rapport peut se transformer au cours de la vie, mais il se constitue beaucoup dans l'enfance. Dans mon rapport au monde, il y a plus de désarroi que de révolte, plus de doutes que de certitudes, beaucoup plus de questions que de réponses. J'aimerais parfois écrire autrement, être plus subversive, plus trash ou plus lyrique, mais je suis ce que je suis. Il faut toute une vie pour accepter que la petite pierre que l'on peut poser, c'est cellelà et pas une autre.

VoIX ET IMAGES Il y a aussi une autre opposition fondamentale dans votre théâtre, celle entre les vraies choses et l'imagination, ce qui est inventé et permet de continuer à vivre, à rêver. Sur ce point, ne croyez-vous pas que les médias jouent le même rôle que le theâtre?

CAROLE FRÉCHETTE Les médias interprètent le réel. Montrer, transmettre les "vraies choses", ça n'existe pas. Dès qu'on les raconte, elles sont transformées. En ce sens, l'information, le documentaire et la fiction ont des points communs. Dans les trois cas, on trafique le réel, mais à des degrés divers, bien sûr. Des reporters nous montrent de «vraies images » qu'ils ont prises sur une scène de guerre, par exemple. Ils les ont choisies parmi des dizaines d'autres, ils les ont assemblées d'une certaine façon, ils les ont commentées. Le tout est présenté dans un topo de deux ou trois minutes, pour une guerre qui s'étend sur tout un pays et dure depuis des mois. Ce n'est pas la vérité, mais une vérité, celle qui est ressentie par le reporter lors de ce jour précis, à ce moment précis. Lorsqu'on est interviewé pour un journal ou à la radio, on passe une ou deux heures avec un journaliste, et au bout du compte, il reste quelques feuillets, quatre ou cinq minutes en ondes. Le réel est constamment réinterprété par les médias, qui essaient de transmettre l'information d'une manière punchée! D'une certaine façon, les journalistes créent de petites fictions. Je ne dis pas que nous faisons le même métier. Pas du tout. Mais nous racontons tous des histoires et nous essayons de les rendre captivantes.

voix ET IMAGES Auteure dramatique, vous avez dû fréquenter les médias, accorder des entrevues, faire la promotion de vos pièces, utiliser des extraits de presse pour présenter des demandes de subventions. L'avez-vous fait à contrecœur? Cela heurtaitil vos principes? Que retenez-vous de cette fréquentation des médias?

CAROLE FRÉCHETTE Je n'ai jamais fait la promotion de mes pièces à contrecœur. Comme tout artiste, j'aime bien que le projecteur soit tourné vers moi. Il faut l'avouer, nous sommes des personnages publics et quand nous mettons nos univers sur une scène, c'est que nous voulons attirer l'attention, sinon nous ne ferions pas ce métier. Je n'ai pas du tout l'impression de me trahir ou de faire des compromis quand je rencontre les journalistes. Par contre, je ne me trouve pas très habile: je suis trop candide. Je ne contrôle pas bien le message que je devrais faire passer pour bien présenter la pièce. Je me laisse porter par les questions, je réponds honnêtement. Cela me joue parfois de mauvais tours. Par exemple, lorsque j'ai été interviewée pour La petite pièce en haut de l'escalier, j'ai déclaré en toute naïveté que c'était, parmi toutes mes pièces, celle que je comprenais le moins. Certains critiques qui n'ont pas aimé le spectacle ont cité ces mots d'une façon qui n'était pas à mon avantage... Je retiens de ma fréquentation des médias qu'il ne faut pas être trop naïf quand on s'adresse à des journalistes, mais je suis certaine que je vais continuer à l'être un peu... Je refuse 
d'aborder ces interviews comme des stratégies de communication dont je devrais savoir tirer toutes les ficelles. Je continue à vouloir avant tout une vraie rencontre avec la personne.

voix ET IMAGES Continuez-vous à croire que les médias peuvent susciter la réflexion et, si oui, à quelles conditions?

CAROLE FRÉCHETte Internet est tellement vaste. On y trouve de tout. On peut certainement y dénicher des faits, des opinions, des réflexions très riches, susceptibles de stimuler notre esprit. La télé est de moins en moins intéressante, il me semble. Elle se standardise et elle est de plus en plus axée sur le divertissement. Il faut que tout y soit joyeux tout le temps. On y trouve de moins en moins d'espace pour «le vrai». La supposée téléréalité trafique complètement le réel. Justement, dans la pièce à laquelle je travaille en ce moment, il est à nouveau question de la télévision et d'Internet. C'est une pièce que j'écris pour un théâtre français, intitulée Small Talk pour le moment. C'est à propos d'une jeune femme qui ne sait pas faire la conversation. Elle en est très malheureuse, car elle se trouve isolée, et elle décide de changer. Elle utilise Internet pour trouver des conseils pratiques. Cette jeune femme a un frère qui, lui, est le roi de la conversation : il anime un talk show à la télé. Un drame se noue autour de cette émission. Il est beaucoup question, dans ce texte, de faux vrai et de vrai faux, de gens ordinaires qu'on essaie de rendre extraordinaires, de vérité, de mensonge, de naturel et d'artificiel.

VOIX ET IMAGES En conclusion, pour vous, dans quelle mesure le théâtre a-t-il un rôle à jouer dans la critique des médias de masse?

CAROLE FRÉCHETte Je ne sais pas si le théâtre doit faire la critique des médias de masse. Par exemple, je n'écris pas la pièce que je suis en train d'écrire pour faire la critique des médias de masse. Cependant, je ne peux faire autrement que de signaler que, dans l'émission de télé que je présente et où l'on se dit très préoccupé du vrai, on ne montre que du faux vrai, que du vrai fabriqué. On trouve une certaine critique des médias dans mes pièces, mais le théâtre est surtout un lieu où l'on peut se rencontrer autrement qu'à travers le prisme des médias. Qu'il parle ou non des médias, le théâtre est important parce qu'il propose un autre type d'échange. C'est un lieu précieux, exigeant, nécessaire, même s'il ne livre pas toujours l'extase attendue. Le théâtre n'est pas dans la course de la cote d'écoute à tout prix. Il peut se permettre d'établir un autre type de contact avec le spectateur, dans une vraie présence de l'un à l'autre, pendant un certain temps. En cela, il est déjà une critique des médias. En tout cas, il est une solution de rechange... même s'il perd de la popularité, ce qui m'inquiète. J'ai toujours cru que le théâtre allait survivre à tout, en raison de son caractère archaïque. Je me disais : on aura toujours besoin de se réunir autour d'une scène illuminée pour se faire raconter une histoire, comme on continuera toujours à se réunir autour d'une table pour manger entre amis. (Est-ce si vrai maintenant? À quand les fêtes d'amis par Skype?) Mais j'avoue que, pour la première fois, je m'interroge sur son avenir. D'une certaine façon, on le pratique encore comme on le faisait chez les Grecs. Il résiste en un sens à toute évolution technologique. Certaines veines du théâtre utilisent beaucoup la technologie - les Robert Lepage de ce monde - , mais, fondamentalement, le théâtre demeure une activité artisanale et accessible à beaucoup de gens. On peut en faire sans avoir beaucoup d'argent. Autrefois, le théâtre avait pour seuls concurrents le cinéma et la 
télévision. Aujourd'hui, il est concurrencé par le fait que tout individu peut avoir accès au monde entier, directement et instantanément, à partir de son écran d'ordinateur. J'espère que nous allons continuer de nous réunir entre êtres humains et de nous regarder dans les yeux, et que nous ne nous laisserons pas complètement «bouffer» par cet écran qui nous rend zombies. 